\title{
Vinogradov's exponential sum over primes
}

\author{
by \\ Xiumin Ren (Jinan)
}

1. Introduction. Exponential sums play an important role in number theory. Non-trivial upper bound estimates for various exponential sums lead to solutions to problems from equi-distribution theory and additive number theory. In this paper, we are concerned with the exponential sum

$$
S_{\beta}(k, x)=\sum_{x<m \leq 2 x} \Lambda(m) e\left(k \alpha m^{\beta}\right),
$$

where $x \geq 2$ and $k \geq 1$ are the main parameters, $\alpha \neq 0$ and $0<\beta<1$ are fixed, $\Lambda(m)$ is the von Mangoldt function, and $e(z)=e^{2 \pi i z}$. We call $S_{\beta}(k, x)$ Vinogradov's exponential sum, since it was first considered by I. M. Vinogradov in the special case $\beta=1 / 2$. Actually he proved (see [10, Chapter III, Section 4, Theorem 6]) that, for $k \leq x^{1 / 10}$,

$$
S_{1 / 2}(k, x) \ll k^{1 / 4} x^{7 / 8+\varepsilon},
$$

where the implied constant may depend on $\alpha$. Iwaniec and Kowalski (see [4, (13.55)]) remarked that the stronger inequality

$$
S_{1 / 2}(1, x) \ll x^{5 / 6} \log ^{4} x
$$

follows from an application of Vaughan's identity. For general $\beta$ and $k$ as in (1.1), Murty and Srinivas [6] proved recently that

$$
S_{\beta}(k, x) \ll k^{1 / 8} x^{(7+\beta) / 8} \log \left(x k^{3}\right) .
$$

They also considered the corresponding exponential sum over integers, and obtained the bound

$$
\sum_{m \leq x} e\left(k m^{\beta}\right) \ll k^{1 / 4} x^{(2+\beta) / 4} \log \left(x k^{3}\right) .
$$

In this direction, we will prove the following result, where the implied constants may depend on $\alpha$ and $\beta$.

2000 Mathematics Subject Classification: 11L07, 11L20, 11 N05.

Supported by the National Natural Science Foundation (grant no. 10571107). 
THEOREM 1.

(i) We have

$$
S_{\beta}(k, x) \ll\left(k^{1 / 2} x^{(1+\beta) / 2}+x^{4 / 5}+k^{-1 / 2} x^{1-\beta / 2}\right) \log ^{A} x,
$$

where $A$ is an absolute positive constant.

(ii) For $\beta \leq 1 / 2$ and $k \leq x^{1 / 2-\beta}$, we have

$$
S_{\beta}(k, x) \ll\left(k^{1 / 10} x^{3 / 4+\beta / 10}+k^{-1 / 2} x^{1-\beta / 2}\right) \log ^{11} x .
$$

(iii) Under the density hypothesis for the Riemann zeta-function, we have

$$
S_{\beta}(k, x) \ll\left(k^{1 / 2} x^{(1+\beta) / 2}+k^{-1 / 2} x^{1-\beta / 2}\right) \log ^{B} x
$$

for some positive constant $B>2$.

For the density hypothesis, the reader is referred to the statement after (2.3). Here we point out that the Riemann hypothesis implies the density hypothesis. Moreover, if the density hypothesis is replaced by the Lindelöf hypothesis in Theorem 1 (iii), the assertion is true but with $\log ^{B} x$ replaced by $x^{\varepsilon}$, where $\varepsilon>0$ is arbitrary (see the Remark after Lemma 2.1).

Theorem 1 with $\beta=1 / 2$ gives, unconditionally,

$$
\begin{aligned}
& S_{1 / 2}(k, x) \ll\left(k^{1 / 2} x^{3 / 4}+x^{4 / 5}\right) \log ^{A} x, \\
& S_{1 / 2}(1, x) \ll x^{4 / 5} \log ^{11} x,
\end{aligned}
$$

and, under the density hypothesis,

$$
S_{1 / 2}(k, x) \ll k^{1 / 2} x^{3 / 4} \log ^{B} x .
$$

Obviously, Theorem 1(i) improves (1.4), while (1.5) and (1.6) improve (1.2) and (1.3), respectively.

The conditional result (1.7) is known to experts. For example, Iwaniec, Luo, and Sarnak [5] studied the exponential sum

$$
T(q, x)=\sum_{\substack{x<n \leq 2 x \\ n \equiv a(\bmod q)}} \Lambda(n) e(2 \sqrt{n} / q),
$$

which, roughly speaking, is $S_{1 / 2}(2, x)$ but with $n$ in the arithmetic progression $n \equiv a(\bmod q)$ with $(a, q)=1$. They pointed out that the estimate $T(q, x) \ll x^{3 / 4+\varepsilon}$ follows from the density hypothesis, and conjectured that (see [5, Hypothesis S, (1.62)])

$$
T(q, x) \ll x^{1 / 2+\varepsilon},
$$

where the implied constant depends only on $\varepsilon$. They were the first to show that the classical $\mathrm{GL}_{1}$ exponential sums such as $T(q, x)$ or $S_{\beta}(k, x)$ are intimately connected to the Riemann hypothesis for $L$-functions of $\mathrm{GL}_{2}$. Actually they proved that (1.8) implies a quasi-Riemann hypothesis for $L(s, f)$, 
i.e., the existence of a zero-free strip, where $f$ is any holomorphic cusp form of integral weight.

Vinogradov was led to $S_{1 / 2}(k, x)$ via his study of

$$
A(\delta, x)=\#\{p \leq x:\{\alpha \sqrt{p}\}<\delta\},
$$

where $0<\delta \leq 1$ and $\alpha>0$ are fixed, and $\{\alpha \sqrt{p}\}$ is the fractional part of $\alpha \sqrt{p}$. Using (1.2), he proved that

$$
A(\delta, x)=\delta \pi(x)+O\left(x^{9 / 10+\varepsilon}\right),
$$

where $\pi(x)$ is the counting function for prime numbers (see [10, Chapter III, Section 4, Theorem 7]). Recall that a sequence $\left(x_{n}\right)$ of numbers in $[0,1]$ is said to be equi-distributed modulo 1 if for any fixed real numbers $0 \leq a<b \leq 1$ we have

$$
\frac{1}{x} \#\left\{n \leq x: a \leq x_{n} \leq b\right\} \rightarrow b-a
$$

as $x \rightarrow \infty$. We deduce from our Theorem 1 the following strong form of equidistribution of $\left\{\alpha p^{\beta}\right\}$, where the length $\delta$ of the interval may vary with $x$.

Theorem 2. Let $\alpha \neq 0,0<\beta<1$, and $0 \leq d<1$. Let $\delta(x)$ be any function such that $0<\delta(x) \leq 1-d$, and let

$$
A_{\beta}(\delta, x)=\#\left\{p \leq x: d \leq\left\{\alpha p^{\beta}\right\} \leq d+\delta(x)\right\} .
$$

Then

$$
A_{\beta}(\delta, x)=\delta(x) \pi(x)+O\left(\left(x^{(2+\beta) / 3}+x^{1-\beta / 2}\right) \log ^{A+1} x\right)
$$

for $x \geq 2$, where the implied constant depends only on $\beta$ and $\alpha$. In particular, the sequence $\left\{\alpha p^{\beta}\right\}$ is equi-distributed modulo 1.

Taking $\beta=1 / m, \alpha=1 / 2, d=0$ and $\delta(x)=1 / 2$, we find that $A_{1 / m}(1 / 2, x)$ is the number of primes which do not exceed $x$ and lie in the intervals $\left[(2 n)^{m},(2 n+1)^{m}\right)$ for integers $n$. Theorem 2 thus gives the following result.

Corollary 3. Fix a positive integer $m \geq 2$. Then the number of primes which do not exceed $x$ and lie in the intervals $\left[(2 n)^{m},(2 n+1)^{m}\right)$ for integers $n$, is

$$
\frac{1}{2} \pi(x)+O\left(x^{1-\theta_{m}} \log ^{A+1} x\right)
$$

where

$$
\theta_{m}= \begin{cases}1 / 6 & \text { if } m=2 \\ 1 / 2 m & \text { if } m \geq 3\end{cases}
$$

and $A$ is the same as in Theorem 1.

In the special case $m=2,(1.10)$ in Corollary 3 reduces to

$$
\frac{1}{2} \pi(x)+O\left(x^{5 / 6+\varepsilon}\right) \text {. }
$$


This improves Vinogradov's result which has the greater error term $O\left(x^{9 / 10+\varepsilon}\right)$ as a consequence of his (1.9).

Vinogradov proved his results by elementary methods; in contrast, we will apply analytic methods to establish Theorem 1. Our main tools are meanvalue estimates of Dirichlet polynomials and classical zero-density estimates for the Riemann zeta-function.

Combined with new ingredients, the idea in the present paper can also be used to investigate exponential sums of the form

$$
\sum_{x<m \leq 2 x} \mu(m) e\left(k \alpha m^{\beta}\right)
$$

where $\mu(m)$ is the Möbius function. This will appear elsewhere.

Different ideas have been used by the author [8] to get new upper bound estimates for the exponential sum

$$
\sum_{x<m \leq 2 x} \Lambda(m) e\left(m^{\kappa} \alpha\right)
$$

where $\kappa$ is a fixed positive integer, and $\alpha \in[0,1]$. This sum is different from (1.1) in two aspects: (i) $\kappa>0$ is an integer, (ii) it has the parameter $\alpha \in[0,1]$ instead of the $k \alpha$ in (1.1). The reader is referred to [8] for an upper bound estimate of (1.12).

\section{Proof of the main results}

Lemma 2.1. Let $r$ be a positive integer and $l=2 r+1$. Suppose that for $T \geq 2$ and $v=0,1$, there exists $C_{r} \geq 0$ such that

$$
\int_{-T}^{T}\left|\zeta^{(v)}(1 / 2+i t)\right|^{2 r} d t \ll T \log ^{C_{r}} T .
$$

Then

$$
S_{\beta}(k, x) \ll\left(k^{1 / 2} x^{(1+\beta) / 2}+x^{3 / 4+1 / 4 l}+k^{-1 / 2} x^{1-\beta / 2}\right) \log ^{D_{r}} x,
$$

where $D_{r}$ is an absolute positive constant depending on $r$, and the implied constant may depend on $\alpha, \beta$ and $r$.

REMARK. If we replace $\log ^{C_{r}} T$ by $T^{\varepsilon}$ in (2.1), the assertion is true but with $\log ^{D_{r}} x$ replaced by $x^{\varepsilon}$. Note that under the Lindelöf hypothesis, one can let $r \rightarrow \infty$, and thus get

$$
S_{\beta}(k, x) \ll x^{\varepsilon}\left(k^{1 / 2} x^{(1+\beta) / 2}+k^{-1 / 2} x^{1-\beta / 2}\right) .
$$

We will prove Lemma 2.1 in Section 3.

Let $N(\sigma, T)$ denote the number of zeroes of the Riemann zeta-function $\zeta(s)$ in the region

$$
0<\Re s \leq \sigma \leq 1, \quad|\Im s| \leq T .
$$


The zero-density theorems state that there exist functions $B(\sigma)$ satisfying $0 \leq B(\sigma) \leq 1$ for $1 / 2 \leq \sigma \leq 1$ such that

$$
N(\sigma, T) \ll T^{B(\sigma)} \log ^{E} T, \quad \text { where } E \geq 0 .
$$

By (1.7) and (1.8) in Huxley [3], the above estimate (2.2) is true for $B(\sigma)=$ $A(\sigma)(1-\sigma)$ and $E=9$, where

$$
A(\sigma)= \begin{cases}3 /(2-\sigma) & \text { if } 1 / 2 \leq \sigma \leq 3 / 4 \\ 12 / 5 & \text { if } 3 / 4<\sigma \leq 1\end{cases}
$$

The density hypothesis states that $(2.2)$ is true for $B(\sigma)=2(1-\sigma)$ and some $E>0$.

LEMMA 2.2. In terms of (2.2), we have

$$
S_{\beta}(k, x) \ll\left(k^{1 / 2} x^{(1+\beta) / 2}+\max _{1 / 2<\sigma \leq 1} k^{B(\sigma)-1 / 2} x^{f(\sigma, \beta)}+k^{-1} x^{1-\beta}\right) \log ^{2+E} x,
$$

where

$$
f(\sigma, \beta)=\sigma+\beta B(\sigma)-\beta / 2,
$$

and the implied constant may depend on $\alpha$ and $\beta$.

The proof of Lemma 2.2 is postponed until the last section of the paper.

With Lemmas 2.1 and 2.2, the proof of Theorem 1 is immediate.

Proof of Theorem 1. (i) The fourth power moment estimate for $\zeta^{(\nu)}(1 / 2+i t)$ reveals that $(2.1)$ is true for $r=2$ and $C_{2}=8$ (see for example [7, Chapter III]). By Lemma 2.1, we get

$$
S_{\beta}(k, x) \ll\left(k^{1 / 2} x^{(1+\beta) / 2}+x^{4 / 5}+k^{-1 / 2} x^{1-\beta / 2}\right) \log ^{A} x,
$$

where $A=D_{2}$ is an absolute positive constant.

(ii) By Lemma 2.2 with $A(\sigma)$ as in (2.3) and $E=9$, we obtain $S_{\beta}(k, x) \ll\left(k^{1 / 2} x^{(1+\beta) / 2}+\max _{1 / 2<\sigma \leq 1} k^{A(\sigma)(1-\sigma)-1 / 2} x^{f(\sigma, \beta)}+k^{-1} x^{1-\beta}\right) \log ^{11} x$, where, by (2.4),

$$
f(\sigma, \beta)=\sigma+\beta A(\sigma)(1-\sigma)-\beta / 2 .
$$

For $3 / 4<\sigma \leq 1$, we have

$$
k^{A(\sigma)(1-\sigma)-1 / 2} x^{f(\sigma, \beta)}=\left(k^{12 / 5} x^{12 \beta / 5-1}\right)^{1-\sigma} k^{-1 / 2} x^{1-\beta / 2} .
$$

Thus for $0<\beta<1$,

$$
\max _{3 / 4<\sigma \leq 1} k^{A(\sigma)(1-\sigma)-1 / 2} x^{f(\sigma, \beta)}=\max \left\{k^{1 / 10} x^{3 / 4+\beta / 10}, k^{-1 / 2} x^{1-\beta / 2}\right\} .
$$

For $1 / 2<\sigma \leq 3 / 4$, we have

$$
k^{A(\sigma)(1-\sigma)-1 / 2} x^{f(\sigma, \beta)}=k^{5 / 2} x^{5 \beta / 2} g(\sigma),
$$

where $g(\sigma)=k^{-3 /(2-\sigma)} x^{-3 \beta /(2-\sigma)+\sigma}$. 
Since

$$
\begin{aligned}
(2-\sigma)^{2} \frac{g^{\prime}(\sigma)}{g(\sigma)} & =-3 \log k+\left((2-\sigma)^{2}-3 \beta\right) \log x \\
& \geq-3 \log k+(25 / 16-3 \beta) \log x
\end{aligned}
$$

we have $g^{\prime}(\sigma) \geq 0$ for $k \leq x^{25 / 48-\beta}$. Hence

$$
\max _{1 / 2 \leq \sigma \leq 3 / 4} k^{A(\sigma)(1-\sigma)-1 / 2} x^{f(\sigma, \beta)}=k^{5 / 2} x^{5 \beta / 2} g(3 / 4)=k^{1 / 10} x^{3 / 4+\beta / 10} .
$$

Therefore, for $0<\beta \leq 1 / 2$ and $k \leq x^{1 / 2-\beta}$, we have

$$
S_{\beta}(k, x) \ll\left(k^{1 / 2} x^{(1+\beta) / 2}+k^{1 / 10} x^{3 / 4+\beta / 10}+k^{-1 / 2} x^{1-\beta / 2}\right) \log ^{11} x .
$$

(iii) By the density hypothesis with $B(\sigma)=2(1-\sigma)$ and some $E>0$ in (2.2), we have

$$
f(\sigma, \beta)=(1-2 \beta) \sigma+3 \beta / 2
$$

in (2.4). Thus

$$
k^{B(\sigma)-1 / 2} x^{f(\sigma, \beta)}=\left(k^{2} x^{2 \beta-1}\right)^{1-\sigma} k^{-1 / 2} x^{1-\beta / 2} .
$$

It follows that

$$
\max _{1 / 2<\sigma \leq 1} k^{B(\sigma)-1 / 2} x^{f(\sigma, \beta)}=\max \left\{k^{1 / 2} x^{(1+\beta) / 2}, k^{-1 / 2} x^{1-\beta / 2}\right\} .
$$

By Lemma 2.2, we get

$$
S_{\beta}(k, x) \ll\left(k^{1 / 2} x^{(1+\beta) / 2}+k^{-1 / 2} x^{1-\beta / 2}\right) \log ^{B} x,
$$

where $B=2+E$.

To prove Theorem 2, we record the following results of Vinogradov (see [10, Chapter III, Section 2, Lemmas 2 and 3]).

LEMMA 2.3. Let $\varrho$ be a positive integer. Let $a, b$ and $\Delta$ be real numbers such that $0<\Delta<1 / 10, \Delta \leq b-a \leq 1-\Delta$. Then there exists a periodic function $\psi(x)$ of period 1 having the following properties:

(1) $\psi(x)=1$ in the interval $a+\Delta / 2 \leq x \leq b-\Delta / 2$,

(2) $0 \leq \psi(x) \leq 1$ in the interval $-\Delta / 2 \leq x \leq a+\Delta / 2$ and $b-\Delta / 2 \leq$ $x \leq b+\Delta / 2$,

(3) $\psi(x)=0$ in the interval $b+\Delta / 2 \leq x \leq 1+a-\Delta / 2$,

(4) $\psi(x)$ can be expanded in a Fourier series of the type

$$
\psi(x)=b-a+\sum_{m=1}^{\infty}\left(g_{m} e(m x)+h_{m} e(-m x)\right),
$$

where $g_{m}$ and $h_{m}$ depend only on $m, a, b, \Delta$, and

$$
\left|g_{m}\right| \leq k_{m}, \quad\left|h_{m}\right| \leq k_{m}, \quad \text { with } k_{m}= \begin{cases}1 / \pi m & \text { if } m \leq \Delta^{-1} \\ (\varrho / \pi m \Delta)^{\varrho} / \pi m & \text { if } m>\Delta^{-1}\end{cases}
$$


Lemma 2.4. Let $\delta_{1}, \ldots, \delta_{Q}$ be numbers in the interval $[0,1]$. Let numbers $\varrho, a, b, \Delta$ and the function $\psi(x)$ satisfy the conditions of Lemma 2.3, and a number $R$ satisfy the condition $R>\Delta Q$. For the sum

$$
U(a, b)=\sum_{s=1}^{Q} \psi\left(\delta_{s}\right)
$$

let

$$
U(a, b)-(b-a) Q \ll R .
$$

For any $\sigma$ such that $0<\sigma \leq 1$, let $A_{\sigma}$ be the number of $\delta_{s}$ satisfying $\delta_{s}<\sigma$. Then

$$
A_{\sigma}=\sigma Q+R_{\sigma}, \quad \text { where } R_{\sigma} \ll R \text {. }
$$

Proof of Theorem 2. In the notation of Lemma 2.3, let $\varrho$ be an arbitrarily large positive integer and

$$
\Delta=\Delta(\beta)=x^{\max \{-\beta / 2,(\beta-1) / 3\}} .
$$

For real numbers $a, b$ satisfying the conditions of Lemma 2.3, let $\psi(x)=$ $\psi(\beta, x)$ be given in the lemma. For prime numbers $p \leq x$, let $\delta_{p}=\left\{\alpha p^{\beta}\right\}$. Consider the sum

$$
U(a, b)=\sum_{p \leq x} \psi\left(\delta_{p}\right)
$$

Then by Lemma 2.3(4), we have

$$
U(a, b)-(b-a) \pi(x) \ll \sum_{k=1}^{\infty} H_{k}(\beta)\left|T_{k}(\beta)\right|,
$$

where

$$
T_{k}(\beta)=\sum_{p \leq x} e\left(k \alpha p^{\beta}\right)
$$

and

$$
H_{k}(\beta)= \begin{cases}1 / k & \text { if } k \leq \Delta^{-1}, \\ 1 / \Delta^{\varrho} k^{1+\varrho} & \text { if } k>\Delta^{-1} .\end{cases}
$$

By partial summation and Theorem 1(i), we easily obtain

$$
T_{k}(\beta) \ll\left(k^{1 / 2} x^{(1+\beta) / 2}+x^{4 / 5}+k^{-1 / 2} x^{1-\beta / 2}\right) \log ^{A} x .
$$

Let $H=\Delta^{-(1+1 / \varrho)}$. By $(2.6),(2.7)$, and the trivial bound $T_{k}(\beta) \ll x$, the right-hand side of (2.5) is 


$$
\begin{aligned}
& \ll \sum_{k=1}^{\Delta^{-1}} \frac{1}{k}\left(k^{1 / 2} x^{(1+\beta) / 2}+x^{4 / 5}+k^{-1 / 2} x^{1-\beta / 2}\right) \log ^{A} x \\
& \quad+\Delta^{-\varrho} \sum_{\Delta^{-1}<k \leq H} \frac{1}{k^{1+\varrho}}\left(k^{1 / 2} x^{(1+\beta) / 2}+x^{4 / 5}+k^{-1 / 2} x^{1-\beta / 2}\right) \log ^{A} x+x(\Delta H)^{-} \varrho \\
& \ll\left(\Delta^{-1 / 2} x^{(1+\beta) / 2}+x^{4 / 5}+x^{1-\beta / 2}\right) \log ^{A+1} x+x \Delta \\
& \ll\left(x^{(2+\beta) / 3}+x^{1-\beta / 2}\right) \log ^{A+1} x .
\end{aligned}
$$

By Lemma 2.4 and noticing that $A_{\beta}(\delta, x)=A_{d+\delta(x)}-A_{d}$, the desired assertion follows immediately.

3. Proof of Lemma 2.1. Let $M \geq 2$. For $j=1, \ldots, 2 l$, let $M_{j}$ be positive numbers such that

$$
2^{-2 l+1} M<M_{1} \cdots M_{2 l} \leq M, \quad 2 M_{l+1}, \ldots, 2 M_{2 l} \leq(2 M)^{1 / l} .
$$

For

$$
a_{j}(m)= \begin{cases}\log m & \text { if } j=1, \\ 1 & \text { if } j=2, \ldots, l, \\ \mu(m) & \text { if } j=l+1, \ldots, 2 l,\end{cases}
$$

we define the following functions of a complex variable $s$ :

$$
f_{j}(s)=\sum_{m \sim M_{j}} \frac{a_{j}(m)}{m^{s}}, \quad F(s)=f_{1}(s) \cdots f_{2 l}(s) .
$$

Here we recall that $m \sim M_{j}$ means $M_{j}<m \leq 2 M_{j}$.

The mean-value estimate below is important.

Lemma 3.1. Let $2 \leq T \leq M^{c}$ where $c \geq 1$. Suppose that (2.1) is true. Then

$$
\int_{-T}^{T}|F(1 / 2+i t)| d t \ll\left(T+T^{1 / 2} M^{1 / 4+1 / 4 l}+M^{1 / 2}\right) \log ^{H_{r}} M,
$$

where $H_{r}$ is a positive constant depending on $r$, and the implied constant depends only on $c, r$ and the implied constant in (2.1).

To prove Lemma 3.1, we quote the following two well known results (see for example [7, Chapter II], and [9, Lemma 3.19]).

LEMmA 3.2. Let $T, N_{0} \geq 1$ and $N \geq 0$. Let $a_{n}$ be any complex numbers. Then

$$
\int_{-T}^{T}\left|\sum_{n=N_{0}}^{N_{0}+N} \frac{a_{n}}{n^{i t}}\right|^{2} d t \ll \sum_{n=N_{0}}^{N_{0}+N}(T+n)\left|a_{n}\right|^{2}
$$


LEMma 3.3. Let $a_{n}$ be complex numbers and let the series $F(s)=$ $\sum_{n=1}^{\infty} a_{n} / n^{s}$ be absolutely convergent for $\sigma=\Re s>\sigma_{a}$. Define

$$
A(x)=\max _{x / 2 \leq n \leq 2 x}\left|a_{n}\right|, \quad x \geq 1 ; \quad B(\sigma)=\sum_{n=1}^{\infty} \frac{\left|a_{n}\right|}{n^{\sigma}}, \quad \sigma>\sigma_{a} .
$$

Let $T \geq 2$. Then for any $s_{0}=\sigma_{0}+i t_{0}$ and $b>0$ with $\sigma_{0}+b>\sigma_{a}$, one has

$$
\sum_{n \leq x} \frac{a_{n}}{n^{s_{0}}}=\frac{1}{2 \pi i} \int_{b-i T}^{b+i T} F\left(s_{0}+s\right) \frac{x^{s}}{s} d s+R(x, T)
$$

where, on writing $\|x\|$ for the distance from $x$ to the nearest integer $N$,

$$
\begin{aligned}
R(x, T) \ll & \frac{x^{b} B\left(b+\sigma_{0}\right)}{T}+x^{1-\sigma_{0}} A(x) \min \left\{1, \frac{\log x}{T}\right\} \\
& +x^{-\sigma_{0}}\left|a_{N}\right| \min \left\{1, \frac{x}{T\|x\|}\right\} .
\end{aligned}
$$

Proof of Lemma 3.1. Without loss of generality, we can assume that $\left\|M_{i}\right\|=1 / 4$ for $i=1, \ldots, l$. We assume further that $M_{1} \geq \cdots \geq M_{l}$, since for other cases the proof is similar.

CASE I: $\prod_{i=1}^{r} M_{i}>M^{1 / 2-1 / 2 l}$. Writing

$$
F_{1}(t)=\prod_{j=1}^{r} f_{j}(1 / 2+i t), \quad F_{2}(t)=\prod_{j=r+1}^{2 l} f_{j}(1 / 2+i t)
$$

we have

$$
F(1 / 2+i t)=F_{1}(t) F_{2}(t) .
$$

By Lemma 3.3 with $T=M^{c}, s_{0}=1 / 2+i t$, and $b=1 / 2+1 / \log M$,

$$
\begin{aligned}
f_{1}(1 / 2+i t)= & -\frac{1}{2 \pi i} \int_{1 / 2+1 / L-i M^{c}}^{1 / 2+1 / L+i M^{c}} \zeta^{\prime}(1 / 2+i t+w) \frac{\left(2 M_{1}\right)^{w}-M_{1}^{w}}{w} d w \\
& +O\left(M^{-1 / 2} L\right),
\end{aligned}
$$

where $L=\log M$. Now we move the integral leftward along the rectangular contour with vertices $\pm i M^{c}, 1 / 2+1 / L \pm i M^{c}$ to the line $\Re w=0$. Note that $\zeta^{\prime}(s)$ has a double pole at $s=1$. Thus the residue at $w=1 / 2-i t$ is

$$
-\frac{\left(2 M_{1}\right)^{1 / 2-i t} \log \left(2 M_{1}\right)-M_{1}^{1 / 2-i t} \log M_{1}}{1 / 2-i t}+\frac{\left(2 M_{1}\right)^{1 / 2-i t}-M_{1}^{1 / 2-i t}}{(1 / 2-i t)^{2}},
$$

which is $\ll M^{1 / 2} L /(1+|t|)$. By the well known convexity bound (see for example $[9, \S 5.1])$,

$$
\zeta^{(v)}(\sigma+i t) \ll(1+|t|)^{(1-\sigma) / 2} \log ^{v+1}(|t|+2) \quad \text { for } 0<\sigma \leq 1,
$$


the contribution from the two horizontal segments from $\pm i M^{c}$ to $1 / 2+1 / L \pm$ $i M^{c}$ is

$$
\ll L^{2} \max _{0 \leq u \leq 1 / 2+1 / L} M^{c(1-(1 / 2+u)) / 2} \frac{M_{1}^{u}}{M^{c}} \ll M^{-1 / 2} L^{2} .
$$

Moreover, on the vertical segment from $-i M^{c}$ to $i M^{c}$, one has

$$
\frac{\left(2 M_{1}\right)^{i v}-M_{1}^{i v}}{i v} \ll \frac{1}{1+|v|} .
$$

Therefore on writing

$$
g_{1}(t)=\int_{-M^{c}}^{M^{c}}\left|\zeta^{\prime}(1 / 2+i t+i v)\right| \frac{d v}{1+|v|}+1
$$

we get

$$
f_{1}(1 / 2+i t) \ll g_{1}(t)+\frac{M_{1}^{1 / 2} L}{1+|t|} .
$$

Similarly, for $j=2, \ldots, r$, we have

where

$$
f_{j}(1 / 2+i t) \ll g_{j}(t)+\frac{M_{j}^{1 / 2} L}{1+|t|},
$$

$$
g_{j}(t)=\int_{-M^{c}}^{M^{c}}|\zeta(1 / 2+i t+i v)| \frac{d v}{1+|v|}+1
$$

Thus

$$
F_{1}(t) \ll \prod_{j=1}^{r}\left\{g_{j}(t)+\frac{M_{j}^{1 / 2} L}{1+|t|}\right\} \ll\left\{1+\frac{\left(M_{1} \cdots M_{r}\right)^{1 / 2} L^{r}}{1+|t|}\right\} \prod_{j=1}^{r} g_{j}(t),
$$

where for all $t>0$, the implied constant depends only on $c$ and $r$.

By definition

$$
F_{2}(t)=\sum_{M_{r+1} \cdots M_{2 l}<m \leq 2^{2 l-r} M_{r+1} \cdots M_{2 l}} \frac{b(m)}{m^{1 / 2+i t}},
$$

where $|b(m)| \leq d_{2 l-r}(m)$, and $d_{s}(m)$ denotes the number of ways of expressing $m$ as a product of $s$ factors. Thus

$$
F_{2}(t) \ll \sum_{M_{r+1} \cdots M_{2 l}<m \leq 2^{2 l-r} M_{r+1} \cdots M_{2 l}} \frac{d_{2 l-r}(m)}{m^{1 / 2}} \ll\left(M_{r+1} \cdots M_{2 l}\right)^{1 / 2} L^{r_{1}},
$$

where $r_{1}$ is a positive constant depending on $r$. This together with (3.4) and (3.6) gives

$$
F(1 / 2+i t) \ll\left\{\left|F_{2}(t)\right|+\frac{M^{1 / 2} L^{r+r_{1}}}{1+|t|}\right\} \prod_{j=1}^{r} g_{j}(t) .
$$


By Hölder's inequality, we get

$$
\begin{aligned}
\int_{-T}^{T}|F(1 / 2+i t)| d t \ll & \left\{\int_{-T}^{T}\left|F_{2}(t)\right|^{2} d t\right\}^{1 / 2}\left\{\prod_{i=1}^{r} \int_{-T}^{T} g_{i}^{2 r}(t) d t\right\}^{1 / 2 r} \\
& +M^{1 / 2} L^{r+r_{1}} \max _{2 \leq X \leq T} X^{-1}\left\{\prod_{i=1}^{r} \int_{-X}^{X} g_{i}^{r}(t) d t\right\}^{1 / r}
\end{aligned}
$$

By Lemma 3.2, (3.7), and in view of $M_{r+1} \cdots M_{2 l} \ll M /\left(M_{1} M_{2} \cdots M_{r}\right)<$ $M^{1 / 2+1 / 2 l}$, we have

$$
\begin{aligned}
\int_{-T}^{T}\left|F_{2}(t)\right|^{2} d t & \ll \sum_{M_{r+1} \cdots M_{2 l}<m \leq 2^{2 l-r} M_{r+1} \cdots M_{2 l}}(T+m) \frac{d_{2 l-r}^{2}(m)}{m} \\
& \ll\left(T+M^{1 / 2+1 / 2 l}\right) L^{r_{2}},
\end{aligned}
$$

where $r_{2}$ is a positive constant depending on $r$.

Let $2 \leq X \leq T$. By (3.5) and Hölder's inequality, we have

$$
\int_{-X}^{X} g_{1}^{2 r}(t) d t \ll(\log X) \int_{-X}^{X} \int_{-M^{c}}^{M^{c}}\left|\zeta^{\prime}(1 / 2+i t+i v)\right|^{2 r} \frac{d v d t}{1+|v|}+X .
$$

Write $\int_{-M^{c}}^{M^{c}}=\int_{-2 X}^{2 X}+\int_{2 X<|v| \leq M^{c}}$. Then the first term on the right splits accordingly into two quantities which we denote by $G_{1}(X)$ and $G_{2}(X)$, respectively. We have

$$
\begin{aligned}
G_{1}(X) & =(\log X) \int_{-2 X}^{2 X} \frac{d v}{1+|v|} \int_{-X+v}^{X+v}\left|\zeta^{\prime}(1 / 2+i w)\right|^{2 r} d w \\
& \ll\left(\log ^{2} X\right) \int_{-3 X}^{3 X}\left|\zeta^{\prime}(1 / 2+i w)\right|^{2 r} d w \ll X \log ^{C_{r}+2} X,
\end{aligned}
$$

by (2.1). To bound $G_{2}(X)$, we let $w=t+v$. Note that $2 X<|v| \leq M^{c}$ and $|t| \leq X$ imply $|v| \geq|w| / 2$ and $X<|w| \leq 2 M^{c}$. It follows that

$$
\begin{aligned}
G_{2}(X) & \ll X(\log X) \int_{X}^{2 M^{c}}\left|\zeta^{\prime}(1 / 2+i w)\right|^{2 r} \frac{d w}{1+|w|} \\
& \ll X\left(\log ^{2} X\right) \max _{X \leq X^{\prime} \leq M^{c}} \frac{1}{X^{\prime}} \int_{-2 X^{\prime}}^{2 X^{\prime}}\left|\zeta^{\prime}(1 / 2+i w)\right|^{2 r} d w \\
& \ll X \log ^{C_{r}+2} M .
\end{aligned}
$$


Thus we obtain

$$
\int_{-X}^{X} g_{1}^{2 r}(t) d t \ll X L^{C_{r}+2}
$$

Similarly, for $i=2, \ldots, r$,

$$
\int_{-X}^{X} g_{i}^{2 r}(t) d t \ll X L^{C_{r}+2}
$$

Moreover, for $i=1, \ldots, r$,

$$
\int_{-X}^{X} g_{i}^{r}(t) d t \ll X^{1 / 2}\left(\int_{-X}^{X} g_{i}^{2 r}(t) d t\right)^{1 / 2} \ll X L^{C_{r} / 2+1}
$$

Putting (3.9)-(3.12) into (3.8), we get

$$
\begin{aligned}
& \int_{-T}^{T}|F(1 / 2+i t)| d t \\
& \quad \ll\left(T+M^{1 / 2+1 / 2 l}\right)^{1 / 2} T^{1 / 2} L^{\left(r_{2}+C_{r}\right) / 2+1}+M^{1 / 2} L^{r+r_{1}+C_{r} / 2+1} \\
& \quad \ll\left(T+T^{1 / 2} M^{1 / 4+1 / 4 l}+M^{1 / 2}\right) L^{r_{3}},
\end{aligned}
$$

where $r_{3}=\max \left\{r_{2} / 2, r+r_{1}\right\}+C_{r} / 2+1$.

CASE II: $\prod_{i=1}^{r} M_{i} \leq M^{1 / 2-1 / 2 l}$. Since $M_{1} \geq \cdots \geq M_{l}$, we have $\prod_{i=1}^{r} M_{i}$ $\geq M_{r}^{r}$, and hence $M_{r} \leq M^{(l-1) / 2 l r}=M^{1 / l}$. Therefore $M_{j} \leq M^{1 / l}$ holds for $j=r, r+1, \ldots, l$, and also for $j=l+1, \ldots, 2 l$, by (3.1). Let $r_{1}$ be the largest integer such that $\prod_{i=1}^{r_{1}} M_{i} \leq M^{1 / 2-1 / 2 l}$. Let $J_{1}=\left\{1, \ldots, r_{1}+1\right\}$, $J_{2}=\left\{r_{1}+2, \ldots, 2 l\right\}$. Then we have

$$
\prod_{j \in J_{1}} M_{j}+\prod_{j \in J_{2}} M_{j} \ll M^{1 / 2+1 / 2 l} .
$$

For $\nu=1,2$, define

$$
F_{\nu}^{*}(s):=\prod_{j \in J_{\nu}} f_{j}(s)=\sum_{n \leq N_{\nu}} \frac{b_{\nu}(n)}{n^{s}},
$$

where $N_{\nu}=\prod_{j \in J_{\nu}}\left(2 M_{j}\right)$ and $b_{\nu}(n) \ll L d_{2 l}(n)$. By Lemma 3.2, we have

$$
\int_{-T}^{T}\left|F_{\nu}^{*}(1 / 2+i t)\right|^{2} d t \ll L^{2} \sum_{n \leq N_{\nu}}(T+n) \frac{d_{2 l}^{2}(n)}{n} \ll\left(T+N_{\nu}\right) L^{r_{4}},
$$

where $r_{4}$ is a positive constant depending on $r$. Cauchy's inequality now 
gives

$$
\begin{aligned}
\int_{-T}^{T}|F(1 / 2+i t)| d t & \ll\left(T+N_{1}\right)^{1 / 2}\left(T+N_{2}\right)^{1 / 2} L^{r_{4}} \\
& \ll\left(T+T^{1 / 2} M^{1 / 4+1 / 4 l}+M^{1 / 2}\right) L^{r_{4}},
\end{aligned}
$$

since $N_{1}+N_{2} \ll M^{1 / 2+1 / 2 l}$ and $N_{1} N_{2} \ll M$. This together with (3.13) finishes the proof of Lemma 3.1 with $H_{r}=\max \left\{r_{3}, r_{4}\right\}$.

Proof of Lemma 2.1. Integrating by parts, we have

$$
\sum_{x<m \leq 2 x} \Lambda(m) e\left(k \alpha m^{\beta}\right)=\int_{x}^{2 x} e\left(k \alpha u^{\beta}\right) d \sum_{x<m \leq u} \Lambda(m) .
$$

Now we apply Heath-Brown's identity (see [2, Lemma 1]) which states that for $m \leq 2 x$,

$$
\Lambda(m)=\sum_{j=1}^{l}(-1)^{j-1}\left(\begin{array}{l}
l \\
j
\end{array}\right) \sum_{\substack{m_{1} \cdots m_{2 j}=m \\
m_{j+1}, \ldots, m_{2 j} \leq(2 x)^{1 / l}}}\left(\log m_{1}\right) \mu\left(m_{j+1}\right) \cdots \mu\left(m_{2 j}\right) .
$$

Then the right hand side of (3.14) becomes a linear combination of $O\left(\log ^{2 l} x\right)$ terms of the form

$$
\int_{x}^{2 x} e\left(k \alpha u^{\beta}\right) d \Sigma(u ; \mathbf{M})
$$

where

$$
\Sigma(u ; \mathbf{M})=\sum_{\substack{m_{1} \sim M_{1} \\ x<m_{1} \cdots m_{2 l} \leq u}} \ldots \sum_{\substack{m_{2 l} \sim M_{2 l} \\ x<}} a_{1}\left(m_{1}\right) \cdots a_{2 l}\left(m_{2 l}\right)
$$

with $a_{j}(m), j=1, \ldots, 2 l$, as in $(3.2)$, and $M_{j}$ positive integers such that (3.1) holds with $M=x$. Here $\mathbf{M}$ is written for the vector $\left(M_{1}, \ldots, M_{2 l}\right)$. Let $f_{j}(s)$ and $F(s)$ be defined by (3.3). Then Lemma 3.3 gives

$$
\Sigma(u ; \mathbf{M})=\frac{1}{2 \pi i} \int_{1+1 / \log x-i T}^{1+1 / \log x+i T} F(s) \frac{u^{s}-x^{s}}{s} d s+R(u, T),
$$

where

$$
R(u, T) \ll \frac{u \log ^{2} u}{T}+(\log u) \min \left\{1, \frac{u}{T\|u\|}\right\} .
$$

We now move the integral along the rectangular contour with vertices $1 / 2 \pm i T, 1+1 / \log x \pm i T$ to the line $\Re s=1 / 2$. Notice that the integrand is regular inside the rectangular region, and the integral on the two horizontal 
segments is

$$
\ll|F(\sigma \pm i T)| \frac{x^{\sigma}}{T} \ll\left(\prod_{j=1}^{2 l} M_{j}^{1-\sigma}\right) \frac{x^{\sigma} \log x}{T} \ll \frac{x \log x}{T} .
$$

Therefore

$$
\Sigma(u ; \mathbf{M})=\frac{1}{2 \pi} \int_{-T}^{T} F(1 / 2+i t) \frac{u^{1 / 2+i t}-x^{1 / 2+i t}}{1 / 2+i t} d t+\frac{x \log x}{T}+R(u, T) .
$$

Without loss of generality, we assume that $\|x\|=1 / 4$. By (3.15), and by the fact that

$$
\int_{x}^{2 x} \min \left\{1, \frac{u}{T\|u\|}\right\} d u \ll \sum_{x<m \leq 2 x} \int_{0}^{1 / 2} \min \left\{1, \frac{m+t}{T t}\right\} d t \ll \frac{x^{2} \log x}{T},
$$

we find

$$
\int_{x}^{2 x} e\left(k \alpha u^{\beta}\right) d R(u, T) \ll\left(1+\beta k|\alpha| x^{\beta}\right) \frac{x \log ^{2} x}{T} \ll 1
$$

on taking

$$
T=T_{0}=\left(1+k|\alpha| x^{\beta}\right) x \log ^{2} x
$$

We thus get

$$
\begin{aligned}
\int_{x}^{2 x} e\left(k \alpha u^{\beta}\right) & d \Sigma(u ; \mathbf{M}) \\
& =\frac{1}{2 \pi} \int_{-T}^{T} F(1 / 2+i t) \int_{x}^{2 x} u^{-1 / 2+i t} e\left(k \alpha u^{\beta}\right) d u d t+O(1) .
\end{aligned}
$$

To bound the main term in (3.18), we write

$$
\int_{x}^{2 x} u^{-1 / 2+i t} e\left(k \alpha u^{\beta}\right) d u=\frac{1}{\beta} \int_{x^{\beta}}^{(2 x)^{\beta}} v^{-1+1 / 2 \beta} e\left(\frac{t}{2 \beta \pi} \log v+k \alpha v\right) d v .
$$

By Lemmas 4.3 and 4.5 in [9], the last integral is

$$
\begin{aligned}
& \ll x^{1 / 2} \min \left\{1, \frac{1}{\min _{x^{\beta} \leq v \leq(2 x)^{\beta}}|t+2 \beta \pi k \alpha v|}, \frac{1}{\sqrt{|t|}}\right\} \\
& \ll x^{1 / 2} \begin{cases}\frac{1}{\sqrt{1+\beta k|\alpha| x^{\beta}}} & \text { if }|t| \leq 4 \beta k \pi|\alpha|(2 x)^{\beta}, \\
\frac{1}{1+|t|} & \text { if } 4 \beta k \pi|\alpha|(2 x)^{\beta}<|t| \leq T_{0} .\end{cases}
\end{aligned}
$$


Thus

$$
\begin{aligned}
\int_{x}^{2 x} e\left(k \alpha u^{\beta}\right) d \Sigma(u ; \mathbf{M}) \ll & \frac{x^{1 / 2}}{\sqrt{1+\beta k|\alpha| x^{\beta}}} \int_{|t| \leq 4 \beta k \pi|\alpha|(2 x)^{\beta}}\left|F\left(\frac{1}{2}+i t\right)\right| d t \\
& +x^{1 / 2} \int_{4 \beta k \pi|\alpha|(2 x)^{\beta}<|t| \leq T_{0}}\left|F\left(\frac{1}{2}+i t\right)\right| \frac{d t}{1+|t|} .
\end{aligned}
$$

By Lemma 3.1, the right-hand side is

$$
\begin{aligned}
& \ll \frac{x^{1 / 2}}{\sqrt{1+\beta k|\alpha| x^{\beta}}}\left(\beta k|\alpha| x^{\beta}+\left(\beta k|\alpha| x^{\beta}\right)^{1 / 2} x^{1 / 4+1 / 4 l}+x^{1 / 2}\right) \log ^{H_{r}} x \\
& \quad+x^{1 / 2} \max _{4 \beta \pi k|\alpha|(2 x)^{\beta} \leq T \leq T_{0}} T^{-1}\left(T+T^{1 / 2} x^{1 / 4+1 / 4 l}+x^{1 / 2}\right) \log ^{H_{r}} x \\
& \ll\left(k^{1 / 2} x^{(1+\beta) / 2}+x^{3 / 4+1 / 4 l}+k^{-1 / 2} x^{1-\beta / 2}\right) \log ^{H_{r}} x,
\end{aligned}
$$

where the implied constant may depend on $\beta$ and $\alpha$. Thus we get

$$
\int_{x}^{2 x} e\left(k \alpha u^{\beta}\right) d \Sigma(u ; \mathbf{M}) \ll\left(k^{1 / 2} x^{(1+\beta) / 2}+x^{3 / 4+1 / 4 l}+k^{-1 / 2} x^{1-\beta / 2}\right) \log ^{H_{r}} x .
$$

Let $D_{r}=H_{r}+2 l$. We finally obtain

$$
\sum_{x<m \leq 2 x} \Lambda(m) e\left(k \alpha m^{\beta}\right) \ll\left(k^{1 / 2} x^{(1+\beta) / 2}+x^{3 / 4+1 / 4 l}+k^{-1 / 2} x^{1-\beta / 2}\right) \log ^{D_{r}} x .
$$

This completes the proof of Lemma 2.1.

4. Proof of Lemma 2.2. By partial integration we have

$$
\sum_{x<m \leq 2 x} \Lambda(m) e\left(k \alpha m^{\beta}\right)=\int_{x}^{2 x} e\left(k \alpha u^{\beta}\right) d \sum_{m \leq u} \Lambda(m) .
$$

We now apply the explicit formula (see [1, Section 17, (9)]):

$$
\sum_{m \leq u} \Lambda(m)=u-\sum_{|\gamma|<T} \frac{u^{\varrho}}{\varrho}-\frac{\zeta^{\prime}(0)}{\zeta(0)}-\frac{1}{2} \log \left(1-u^{-2}\right)+R(u, T),
$$

where $R(u, T)$ satisfies (3.15) and $\varrho$ denotes a non-trivial zero of $\zeta(s)$ with imaginary part $\gamma$ in the rectangular region $0<\Re s<1,|\Im s| \leq T$. Thus

$$
\begin{aligned}
\sum_{x<m \leq 2 x} \Lambda(m) e\left(k \alpha m^{\beta}\right)= & \int_{x}^{2 x} e\left(k \alpha u^{\beta}\right) d u-\sum_{|\gamma|<T} \int_{x}^{2 x} u^{\varrho-1} e\left(k \alpha u^{\beta}\right) d u \\
& -\int_{x}^{2 x} \frac{e\left(k \alpha u^{\beta}\right)}{u\left(u^{2}-1\right)} d u+\int_{x}^{2 x} e\left(k \alpha u^{\beta}\right) d R(u, T) .
\end{aligned}
$$


Let $T=T_{0}$ be as in (3.17). Then by (3.16), the last term is $O(1)$. Moreover we have

$$
\int_{x}^{2 x} e\left(k \alpha u^{\beta}\right) d u=\frac{1}{\beta} \int_{x^{\beta}}^{(2 x)^{\beta}} u^{1 / \beta-1} e(k \alpha u) d u \ll k^{-1} x^{1-\beta},
$$

and

$$
\int_{x}^{2 x} \frac{e\left(k \alpha u^{\beta}\right)}{u\left(u^{2}-1\right)} d u \ll x^{-2}
$$

Consequently,

$$
\sum_{x<m \leq 2 x} \Lambda(m) e\left(k \alpha m^{\beta}\right)=-\sum_{|\gamma|<T_{0}} \int_{x}^{2 x} u^{\varrho-1} e\left(k \alpha u^{\beta}\right) d u+O\left(1+k^{-1} x^{1-\beta}\right) .
$$

By Lemmas 4.3 and 4.5 in [9], and similarly to (3.19) and (3.20), we have

$$
\int_{x}^{2 x} u^{\varrho-1} e\left(k \alpha u^{\beta}\right) d u \ll x^{\sigma} \begin{cases}\frac{1}{\sqrt{1+\beta k|\alpha| x^{\beta}}} & \text { if }|\gamma| \leq 4 \beta k \pi|\alpha|(2 x)^{\beta}, \\ \frac{1}{1+|\gamma|} & \text { if } 4 \beta k \pi|\alpha|(2 x)^{\beta}<|\gamma|<T_{0} .\end{cases}
$$

Therefore,

$$
\begin{aligned}
\sum_{|\gamma|<T_{0}} \int_{x}^{2 x} u^{\varrho-1} e\left(k \alpha u^{\beta}\right) d u \ll & \frac{1}{\left(1+k|\alpha| x^{\beta}\right)^{1 / 2}} \sum_{|\gamma| \leq 4 \beta k \pi|\alpha|(2 x)^{\beta}} x^{\sigma} \\
& +\sum_{4 \beta k \pi|\alpha|(2 x)^{\beta}<|\gamma|<T_{0}} \frac{x^{\sigma}}{1+|\gamma|} .
\end{aligned}
$$

Integrating by parts and applying (2.2), we have, for $t \geq 2$,

$$
\sum_{|\gamma| \leq t} x^{\sigma}=-\int_{0}^{1} x^{\sigma} d N(\sigma, t) \ll(\log t)\left(x^{1 / 2} t+\left(\log ^{E} t\right) \max _{1 / 2 \leq \sigma \leq 1} t^{B(\sigma)} x^{\sigma}\right) .
$$

Here we have used the fact that $N(\sigma, t) \ll t \log t$. Thus the first term in the right-hand side of (4.1) is

$$
\ll\left(\log ^{1+E} x\right)\left(k^{1 / 2} x^{(1+\beta) / 2}+\max _{1 / 2 \leq \sigma \leq 1} k^{B(\sigma)-1 / 2} x^{\sigma+\beta B(\sigma)-\beta / 2}\right) .
$$

By (4.2) and in view of $B(\sigma) \leq 1$, we also have

$$
\begin{aligned}
\sum_{4 \beta k \pi|\alpha|(2 x)^{\beta}<|\gamma|<T_{0}} \frac{x^{\sigma}}{1+|\gamma|} & \ll(\log x) \max _{4 \beta k \pi|\alpha|(2 x)^{\beta} \leq t \leq T_{0}} t^{-1} \sum_{|\gamma| \sim t} x^{\sigma} \\
& \ll\left(\log ^{2+E} x\right)\left(x^{1 / 2}+\max _{1 / 2 \leq \sigma \leq 1} k^{B(\sigma)-1} x^{\sigma+\beta B(\sigma)-\beta}\right) .
\end{aligned}
$$


Let $f(\sigma, \beta)$ be as in (2.4). We can conclude that

$$
\begin{aligned}
& \sum_{x<m \leq 2 x} \Lambda(m) e\left(k \alpha m^{\beta}\right) \\
& \quad \ll\left(\log ^{2+E} x\right)\left(k^{1 / 2} x^{(1+\beta) / 2}+\max _{1 / 2 \leq \sigma \leq 1} k^{B(\sigma)-1 / 2} x^{f(\sigma, \beta)}+k^{-1} x^{1-\beta}\right),
\end{aligned}
$$

where the implied constant depends on $\alpha$ and $\beta$.

Acknowledgements. The author is grateful to the referee for valuable suggestions that clarify the argument of the paper and for suggesting the study of the exponential sum (1.11).

\section{References}

[1] H. Davenport, Multiplicative Number Theory, 2nd ed., Springer, Berlin, 1980.

[2] D. R. Heath-Brown, Prime numbers in short intervals and a generalized Vaughan's identity, Canad. J. Math. 34 (1982), 1365-1377.

[3] M. N. Huxley, On the difference between consecutive primes, Invent. Math. 15 (1972), 164-170.

[4] H. Iwaniec and E. Kowalski, Analytic Number Theory, Amer. Math. Soc. Colloq. Publ. 53, Amer. Math. Soc., Providence, RI, 2004.

[5] H. Iwaniec, W. Luo and P. Sarnak, Low lying zeros of families of L-functions, Inst. Hautes Études Sci. Publ. Math. 91 (2000), 55-131.

[6] M. R. Murty and K. Srinivas, On the uniform distribution of certain sequences, Ramanujan J. 7 (2003), 185-192.

[7] C. D. Pan and C. B. Pan, Goldbach Conjecture, Science Press, Beijing, 1992.

[8] X. M. Ren, On exponential sums over primes and application in Waring-Goldbach problem, Sci. China Ser. A 48 (2005), 785-797.

[9] E. C. Titchmarsh, The Theory of the Riemann Zeta-Function, 2nd ed., Oxford Univ. Press, Oxford, 1986.

[10] I. M. Vinogradov, Special Variants of the Method of Trigonometric Sums, Nauka, Moscow, 1976 (in Russian); English transl.: I. M. Vinogradov, Selected Works, Springer, Berlin, 1985.

Department of Mathematics

Shandong University

Jinan, Shandong 250100, P.R. China

E-mail: xmren@sdu.edu.cn

Received on 19.9.2005

and in revised form on 18.4.2006 